\title{
Bayesian Rendezvous for Distributed Robotic Systems
}

\author{
Sven Gowal and Alcherio Martinoli
}

\begin{abstract}
In this paper, we state, using thorough mathematical analysis, sufficient conditions to perform a rendezvous maneuver with a group of differential-wheeled robots endowed with an on-board, noisy, local positioning system. In particular, we extend the existing framework of noise-free, graph-based distributed control with a layer of Bayesian reasoning allowing to solve the rendezvous problem more efficiently in presence of uncertainties and in a probabilistically sound way. Finally we perform extensive experiments with a team of four real robots, and simulation with their corresponding simulated counterpart, to confirm the benefits of our Bayesian approach.
\end{abstract}

\section{INTRODUCTION}

Since the 1960s, consensus problems have puzzled the minds of many researchers in various fields, ranging from computer science to information aggregation [16]. The term consensus describes the problem of reaching an agreement amongst different agents on a certain quantity or state. These agents can share information about their state either by means of communication or observations. In a network of robots, solving the consensus problem on the position of each agents refers to the task of controlling them as to reach a common rendezvous point. To the best of our knowledge, the first occurence of rendezvous for mobile agents was introduced by Reynolds [20] in 1987 as one of the three rules stated to create a flocking behavior, known as the flock centering rule.

It is only much later in 1999 that convergence of mobile robots to a common location in space was studied by Ando et al. [1]. It was then extended for both synchronous and asynchronous cases by Lin at al. $[13,14]$. However, they consider a simple version of the rendezvous problem where the mobile robots are holonomic (i.e. they are capable of moving in any direction at any time), thus yielding simpler control laws and tractable convergence properties. Other methods used to achieve such a holonomic rendezvous include Laplacian feedback [8, 10], cyclic pursuit [21], potential fields [12] or even curve shortening [24]. Solving the rendezvous with nonholonomic agents is more complex and proving the convergence property can be difficult. Many work employ feedback linearization to design relaxed control laws that recreate the holonomic properties [11, 19]. Other works create algorithms that are very specific to their application needs $[4,5]$. But most of them rely on deterministic assumptions and rarely incorporate stochasticity as a modus operandi.

On another front, probabilistic consensus or the idea of reaching an agreement when observations are noisy received

Sven Gowal and Alcherio Martinoli are with the Distributed Intelligent Systems and Algorithms Laboratory, School of Architecture, Civil and Environmental Engineering, Ecole Polytechnique Fédérale de Lausanne. svenadrian.gowaldepfl.ch, alcherio.martinolidepfl.ch

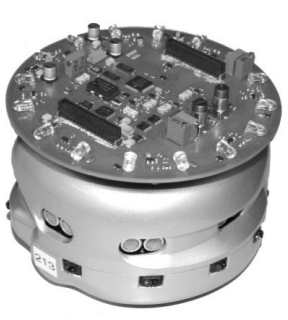

(a)

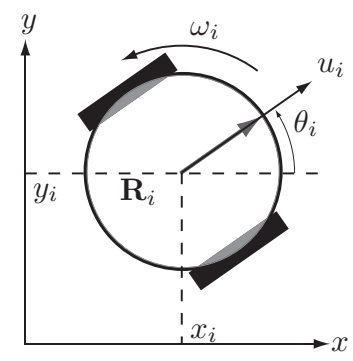

(b)
Fig. 1. (a) A Khepera III robot with a range and bearing module attached. This range and bearing platform features sixteen infrared light emitting diodes and eight infrared light sensors. (b) The kinematic model of a differential-wheeled robot $\mathbf{R}_{i}$.

attention for a long time, indeed, from 1959 with Eisenberg and Gale [6] to 2008 with Cao et al. [3]. However, no contribution focused on the rendezvous problem on non-holonomic agents capable of performing noisy positioning observations neither form a theoretical nor form an experimental point of view.

\section{A. Related Work}

Using limited perception or localization capabilities to create robotic formations or acheive the rendezvous has received recent attention in $[22,23]$. The ability to leverage these limitations on non-holonomic robots to create static configurations of robots is expressed inter alia by the suite of papers [4, 5, 7]. Unfortunately, these contributions treat the rendezvous problem in a deterministic fashion and are therefore not applicable to platforms whose sensory performance is affected by noise and further limitations in terms of range and update speed. Other work such as [2] study the convergence properties of holonomic agents under noisy measurements in simulation. In this work, we will build upon [7] a layer of Bayesian reasoning allowing for a probabilistically sound convergence of real non-holonomic agents, namely the Khepera III robot [17], equipped with a relative range and bearing module [18] (see Figure 1(a)), delivering noisy local positioning measurements.

\section{B. Problem Statement}

Let us assume we have a team of $N$ differential-wheeled robots $\mathbf{R}_{1}, \ldots, \mathbf{R}_{N}$ driven by the following kinematic equations:

$$
\left\{\begin{array}{l}
\dot{x}_{i}=u_{i} \cos \theta_{i} \\
\dot{y}_{i}=u_{i} \sin \theta_{i} \\
\dot{\theta}_{i}=\omega_{i}
\end{array}\right.
$$

where $u_{i}$ is the linear translational speed, $\omega_{i}$ the rotational speed and the vector $\left[x_{i}, y_{i}, \theta_{i}\right]^{\top}$ forms the triplet defining the absolute pose or state of the robot $\mathbf{R}_{i}$, as shown on Figure 1(b). Additionally, each robot $\mathbf{R}_{i}$ has a set of neighbors 


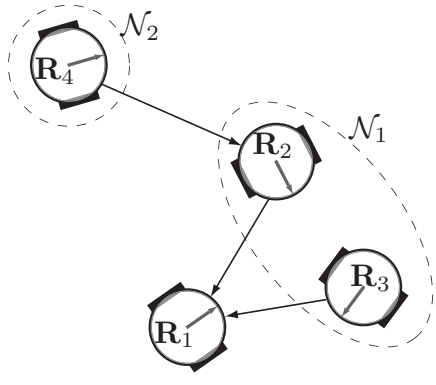

(a)

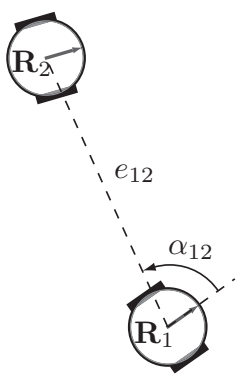

(b)
Fig. 2. (a) Robots $\mathbf{R}_{2}$ and $\mathbf{R}_{3}$ are in the neighborhood of robot $\mathbf{R}_{1}$, i.e. $\mathcal{N}_{1}=\left\{\mathbf{R}_{2}, \mathbf{R}_{3}\right\}$, and robot $\mathbf{R}_{4}$ is the only neighbor of robot $\mathbf{R}_{2}$, i.e. $\mathcal{N}_{2}=\left\{\mathbf{R}_{4}\right\}$. An arrow indicates that the robot at its head can observe the robot at its tail. (b) The range $e_{i j}$ between two robots is the Euclidean distance $\sqrt{\left(x_{j}-x_{i}\right)^{2}+\left(y_{j}-y_{i}\right)^{2}}$ separating both robot's centers and the bearing $\alpha_{i j}$ is the angle between the forward vector $\left[\cos \theta_{i}, \sin \theta_{i}\right]^{\top}$ and difference vector $\left[x_{j}-x_{i}, y_{j}-y_{i}\right]^{\top}$.

$\mathcal{N}_{i}$ containing all robots $\mathbf{R}_{j}$ such that it can measure the range $e_{i j}$ and bearing $\alpha_{i j}$ to them. Its measurements of the range and bearing may be affected by noise such that each observation $z_{i j}(t)$ of $\mathbf{R}_{j}$ at time $t$ is defined by the vector

$$
z_{i j}(t)=\left[\begin{array}{c}
\tilde{e}_{i j}(t) \\
\tilde{\alpha}_{i j}(t)
\end{array}\right]=\left[\begin{array}{c}
e_{i j}(t) \\
\alpha_{i j}(t)
\end{array}\right]+\epsilon_{z}
$$

where $\epsilon_{z}$ is a random noise vector sampled for each observation from a probability distribution given by its probability density function $\mathbf{p d f} \mathbf{f}_{z}(\epsilon)$. Hence at time $t$, a robot $\mathbf{R}_{i}$ gathers an observation list $Z_{i}(t)=\left\{z_{i j}(t) \mid \mathbf{R}_{j} \in \mathcal{N}_{i}\right\}$. Our goal is to provide a control law that incorporates our knowledge of $\mathbf{p d f}_{z}(\epsilon)$ to drive the $N$ robots to a common rendezvous point. This description is schematized on Figure 2. Finally it is useful to introduce the following notation to gather all distances and bearings between a robot $\mathbf{R}_{i}$ and its neighbors: $\triangleright \mathbf{e}_{i}=\left[e_{1}, \ldots, e_{k}, \ldots, e_{\left|\mathcal{N}_{i}\right|}\right]^{\top}$ with $e_{k}=e_{i j}$ $\triangleright \boldsymbol{\alpha}_{i}=\left[\alpha_{1}, \ldots, \alpha_{k}, \ldots, \alpha_{\left|\mathcal{N}_{i}\right|}\right]^{\top}$ with $\alpha_{k}=\alpha_{i j}$

for each robot $\mathbf{R}_{j} \in \mathcal{N}_{i}$ and their corresponding observations:

$\triangleright \tilde{\mathbf{e}}_{i}=\left[\tilde{e}_{1}, \ldots, \tilde{e}_{k}, \ldots, \tilde{e}_{\left|\mathcal{N}_{i}\right|}\right]^{\top}$ with $\tilde{e}_{k}=\tilde{e}_{i j}$

$\triangleright \tilde{\boldsymbol{\alpha}}_{i}=\left[\tilde{\alpha}_{1}, \ldots, \tilde{\alpha}_{k}, \ldots, \tilde{\alpha}_{\left|\mathcal{N}_{i}\right|}\right]^{\top}$ with $\tilde{\alpha}_{k}=\tilde{\alpha}_{i j}$

for each robot $\mathbf{R}_{j} \in \mathcal{N}_{i}$.

\section{A. Graph Theory}

\section{PRELIMINARIES}

Our network of robots can be seen as a graph containing $N$ elements and can be described by the tuple $\mathcal{G}=\{\mathcal{V}, \mathcal{E}\}$, where

$\triangleright \mathcal{V}=\left\{\mathbf{R}_{1}, \ldots, \mathbf{R}_{N}\right\}$ is the vertex set and

$\triangleright \mathcal{E}=\left\{\mathrm{e}_{k} \mid \mathrm{e}_{k}=\left(\mathbf{R}_{i}, \mathbf{R}_{j}\right) \Longrightarrow \mathbf{R}_{j} \in \mathcal{N}_{i}\right\}$ is the edge set.

A graph is said to be strongly connected if there exists a path from any vertex to any other vertex in the graph. It is weakly connected if by adding for each edge $\left(\mathbf{R}_{i}, \mathbf{R}_{j}\right)$ a new edge $\left(\mathbf{R}_{j}, \mathbf{R}_{i}\right)$ to the edge set yields a strongly connected graph.

On a graph, one can define an adjacency matrix $\mathcal{A}$ such that each of its element $a_{i j}$ is defined as

$$
a_{i j}=\left\{\begin{array}{ll}
1 & \text { if } \mathrm{e}_{k}=\left(\mathbf{R}_{i}, \mathbf{R}_{j}\right) \in \mathcal{E} \\
0 & \text { otherwise }
\end{array} .\right.
$$

A graph is symmetric if $a_{i j}=a_{j i}$. Note that a symmetric and weakly connected graph is by definition strongly connected.

\section{B. Noise-free convergence}

Theorem 1: Given a symmetric and weakly connected group of $N$ differential-wheeled robots $\mathbf{R}_{1}, \ldots, \mathbf{R}_{N}$ driven by the kinematic Equation 1, the control law

$$
\left\{\begin{array}{l}
u_{i}=k_{u}\left(t, \mathbf{e}_{i}, \boldsymbol{\alpha}_{i}\right) \sum_{\mathbf{R}_{j} \in \mathcal{N}_{i}} e_{i j} \cos \alpha_{i j} \\
\omega_{i}=k_{\omega}\left(t, \mathbf{e}_{i}, \boldsymbol{\alpha}_{i}\right)
\end{array}\right.
$$

where $k_{u}(\cdot)>0$ for all $t \geq 0$, drives the group to a common rendezvous point if and only if $u_{i}=0$ and $e_{i j} \neq 0$ imply that $k_{\omega}(\cdot) \neq 0$ for at least one pair of robots $\mathbf{R}_{i}$ and $\mathbf{R}_{j}$.

Proof: Let us assume that our group of $N$ robots is connected by an underlying graph defined by the adjacency matrix $\mathcal{A}=\mathcal{A}^{\top}$ (symmetric graph). The notion of symmetric connection in a group of homogeneous robots does make sense in reality where, often, if a robot $\mathbf{R}_{i}$ can observe another robot $\mathbf{R}_{j}$ then $\mathbf{R}_{j}$ can observe $\mathbf{R}_{i}$. Each robot $\mathbf{R}_{i}$ can use the range $e_{i j}$ and bearing $\alpha_{i j}$ to each of its neighbors $\mathbf{R}_{j}$ to steer the whole group towards a single meeting point. As done in [4], we can define a candidate Lyapunov function

$$
V(\mathbf{e})=\sum_{i=1}^{N} \sum_{\mathbf{R}_{j} \in \mathcal{N}_{i}} e_{i j}^{2}=\sum_{i=1}^{N} \sum_{j=1}^{N} a_{i j} e_{i j}^{2}
$$

with $\mathbf{e}=\left[e_{1}, \ldots, e_{|\mathcal{E}|}\right]^{\top}$ with $e_{k}=e_{i j}$ for each edge $\mathrm{e}_{k}=\left(\mathbf{R}_{i}, \mathbf{R}_{j}\right) \in \mathcal{E}$. It is clear that $V(\mathbf{0})=0$ when all inter-neighbor distances $e_{i j}$ are 0 , for all $\mathbf{R}_{i}, \mathbf{R}_{j} \in \mathcal{N}_{i}$ and $V(\mathbf{e})>0$ otherwise. Hence according to Lyapunov's second theorem, if $V$ is a valid Lyapunov function and the graph is at least weakly connected then all robots should converge to the same location (independently of the graph topology). If the graph is disconnected, then only robots connected to each other reach the same location in space. Note that

$$
e_{i j}=\sqrt{\left(x_{j}-x_{i}\right)^{2}+\left(y_{j}-y_{i}\right)^{2}}
$$

and combining it with Equation 1 yields

$$
\dot{e}_{i j}=-u_{i} \cos \alpha_{i j}-u_{j} \cos \alpha_{j i} .
$$

The derivative of $V$ with respect to time becomes

$$
\begin{aligned}
\dot{V}(\mathbf{e}) & =2 \sum_{i=1}^{N} \sum_{j=1}^{N} a_{i j} e_{i j} \dot{e}_{i j} \\
& =-2 \sum_{i=1}^{N} \sum_{j=1}^{N} a_{i j} u_{i} e_{i j} \cos \alpha_{i j}+a_{i j} u_{j} e_{i j} \cos \alpha_{j i} \\
& =-2 \sum_{i=1}^{N} \sum_{j=1}^{N} a_{i j} u_{i} e_{i j} \cos \alpha_{i j}+\sum_{j=1}^{N} a_{j i} u_{i} e_{j i} \cos \alpha_{i j}
\end{aligned}
$$

Since $\mathcal{A}$ is symmetric $\left(a_{i j}=a_{j i}\right)$ and $e_{i j}=e_{j i}$, we obtain

$$
\begin{aligned}
\dot{V}(\mathbf{e}) & =-4 \sum_{i=1}^{N} \sum_{j=1}^{N} a_{i j} u_{i} e_{i j} \cos \alpha_{i j} \\
& =-4 \sum_{i=1}^{N} u_{i} \sum_{j=1}^{N} a_{i j} e_{i j} \cos \alpha_{i j}
\end{aligned}
$$




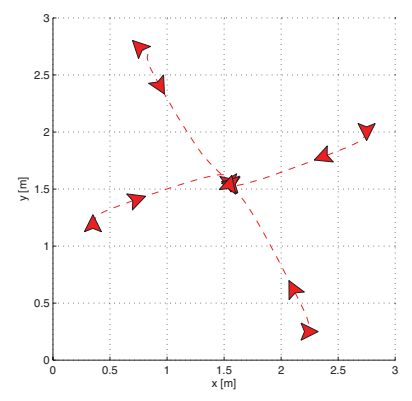

(a)

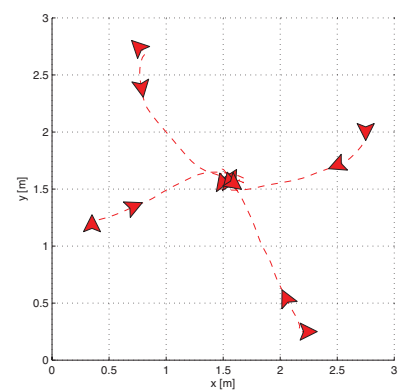

(b)
Fig. 3. Four robots converge to a common meeting point (a) without and (b) with the presence of noise using Equation 6 and 10. Here we have used $K_{u}=1 / 2$ and $K_{\omega}=2$. Additionally we have assumed an independent noise $\epsilon_{z}=\left[\epsilon_{e}, \epsilon_{\alpha}\right]^{\top}$ with $\epsilon_{e} \sim \mathcal{N}\left(0,0.05^{2}\left[\mathrm{~m}^{2}\right]\right)$ and $\epsilon_{\alpha} \sim \mathcal{N}\left(0,0.15^{2}\left[\operatorname{rad}^{2}\right]\right)$ and an average time interval between observations steps of $d t=0.2[\mathrm{~s}]$.

Posing

$$
u_{i}=k_{u}\left(t, \mathbf{e}_{i}, \boldsymbol{\alpha}_{i}\right) \sum_{j=1}^{N} a_{i j} e_{i j} \cos \alpha_{i j}
$$

finally results in

$$
\begin{aligned}
\dot{V}(\mathbf{e}) & =-4 \sum_{i=1}^{N} k_{u}\left(t, \mathbf{e}_{i}, \boldsymbol{\alpha}_{i}\right)\left(\sum_{j=1}^{N} a_{i j} e_{i j} \cos \alpha_{i j}\right)^{2} \\
& \leq 0 .
\end{aligned}
$$

We observe that $V(\mathbf{0})=\dot{V}(\mathbf{0})=0$. Unfortunately, $\dot{V}(\mathbf{e})=0$ even when $\mathbf{e} \neq \mathbf{0}$, hence $\dot{V}$ is only negative semi-definite. However, the set $\mathcal{S}=\{\mathbf{e} \mid \dot{V}(\mathbf{e})=0\}$ does not contain any trajectories of the system, except the trivial trajectory e $(t)=$ $\mathbf{0}, \forall t$. Indeed $\mathcal{S}$ is equivalent to $\left\{\mathbf{e} \mid u_{i}=0, \forall i\right\}$ and we know that

$$
\begin{aligned}
& \alpha_{i j}=\operatorname{atan} 2\left(y_{j}-y_{i}, x_{j}-x_{i}\right)-\theta_{i} \text { and thus } \\
& \dot{\alpha}_{i j}=\frac{u_{i} \sin \alpha_{i j}+u_{j} \sin \alpha_{j i}}{e_{i j}}-\omega_{i} .
\end{aligned}
$$

Hence, inside the set of trajectories $\mathcal{S}$ we have $\dot{\alpha}_{i j}=-\omega_{i}$. As we have stated that at least one robot $\mathbf{R}_{i}$ such that $e_{i j} \neq 0$ for a neighboring $\mathbf{R}_{j}$ will have a rotational speed $\omega_{i} \neq 0, u_{i}$ will change and become different than 0 yielding a $\dot{V}(\mathbf{e})<0$. Therefore according to the Krasovskii-LaSalle principle, we conclude that the system of robots converges asymptotically to a common point in space.

As an example, let us use

$$
\left\{\begin{array}{l}
u_{i}=K_{u} \sum_{\mathbf{R}_{j} \in \mathcal{N}_{i}} e_{i j} \cos \alpha_{i j} \\
\omega_{i}=K_{\omega} \sum_{\mathbf{R}_{j} \in \mathcal{N}_{i}} \sin \alpha_{i j}
\end{array}\right.
$$

with $K_{u}$ and $K_{\omega}$ two positive constants. It is easy to verify that, according to Theorem 1, this control law drives the group to a common rendezvous point. Figure 3(a) shows a simulation run with four robots using this control law and how they converge to a single rendezvous location.

\section{NOISY MEASUREMENTS}

Theorem 2: Given a symmetric and weakly connected group of $N$ differential-wheeled robots $\mathbf{R}_{1}, \ldots, \mathbf{R}_{N}$ driven by the kinematic Equation 1, the control law depending only on noisy measurements $\tilde{e}_{i j}$ and $\tilde{\alpha}_{i j}$ given by Equation 2:

$$
\left\{\begin{array}{l}
\tilde{u}_{i}=k_{u}\left(t, \tilde{\mathbf{e}}_{i}, \tilde{\boldsymbol{\alpha}}_{i}\right) \sum_{\mathbf{R}_{j} \in \mathcal{N}_{i}} \tilde{e}_{i j} \cos \tilde{\alpha}_{i j} \\
\tilde{\omega}_{i}=k_{\omega}\left(t, \tilde{\mathbf{e}}_{i}, \tilde{\boldsymbol{\alpha}}_{i}\right)
\end{array}\right.
$$

where $k_{u}(\cdot)>0$ for all $t \geq 0$, drives the group almost surely to a common rendezvous point if and only if $(i) \tilde{u}_{i}=0$ and $\tilde{e}_{i j} \neq 0$ imply that $k_{\omega}(\cdot) \neq 0$ for at least one pair of robots $\mathbf{R}_{i}$ and $\mathbf{R}_{j}$ and (ii) the expected value $\mathbb{E}_{\epsilon_{z}}\left[\tilde{u}_{i}\right]$ of $\tilde{u}_{i}$ over the observation noise which depends solely on the probability density function $\mathbf{p d f}_{z}(\epsilon)$ has the same sign than its equivalent deterministic control $u_{i}$ as given by Equation 3 (i.e. $\mathbb{E}_{\epsilon_{z}}\left[\tilde{u}_{i}\right] \cdot u_{i} \geq 0$ ).

Remark 1: The first condition $(i)$ on $k_{\omega}(\cdot)$ may be safely ignored if the noise component $\epsilon_{z}$ may take values different than zero.

Proof: Let us initially take a detour by assuming that our dynamical system of robots is discrete. At each time step lasting $d t$ seconds, each robot moves according to an Euler integration of its kinematic model given by Equation 1:

$$
\left\{\begin{array}{l}
x_{i}(t+d t)=\tilde{u}_{i} \cos \theta_{i} \cdot d t+x_{i} \\
y_{i}(t+d t)=\tilde{u}_{i} \sin \theta_{i} \cdot d t+y_{i} \\
\theta_{i}(t+d t)=\tilde{\omega}_{i} \cdot d t+\theta_{i}
\end{array}\right.
$$

where $x_{i} \equiv x_{i}(t), y_{i} \equiv y_{i}(t), \theta_{i} \equiv \theta_{i}(t), \tilde{u}_{i} \equiv \tilde{u}_{i}(t)$ and $\tilde{\omega}_{i} \equiv \tilde{\omega}_{i}(t)$. We can then define a stochastic Lyapunov candidate function as in Equation 4:

$$
V(t)=\sum_{i=1}^{N} \sum_{j=1}^{N} a_{i j} e_{i j}^{2}(t)
$$

and find the difference $\Delta V(t)=V(t+d t)-V(t)$ between the values of that function at two consecutive time steps. Using the relation in Equation 5 and additional trigonometric properties, we obtain

$$
\begin{aligned}
\Delta V(t) & =V(t+d t)-V(t) \\
& =\sum_{i=1}^{N} \sum_{j=1}^{N} a_{i j}\left(e_{i j}^{2}(t+d t)-e_{i j}^{2}(t)\right) \\
& =-2 d t \cdot \sum_{i=1}^{N} \sum_{j=1}^{N} a_{i j}\left(\tilde{u}_{i} e_{i j} \cos \alpha_{i j}+\tilde{u}_{j} e_{i j} \cos \alpha_{j i}\right) \\
& +d t^{2} \cdot \sum_{i=1}^{N} \sum_{j=1}^{N} a_{i j}\left(\tilde{u}_{i}^{2}+\tilde{u}_{j}^{2}+2 \tilde{u}_{i} \tilde{u}_{j} \cos \left(\alpha_{i j}-\alpha_{j i}\right)\right) .
\end{aligned}
$$

Given the symmetry of $\mathcal{A}, \Delta V(t)$ becomes

$$
\begin{aligned}
\Delta V(t) & =-4 d t \cdot \sum_{i=1}^{N} \tilde{u}_{i} \sum_{j=1}^{N} a_{i j} e_{i j} \cos \alpha_{i j} \\
& +2 d t^{2} \cdot \sum_{i=1}^{N}\left(\left|\mathcal{N}_{i}\right| \tilde{u}_{i}^{2}+\tilde{u}_{i} \sum_{j=1}^{N} a_{i j} \tilde{u}_{j} \cos \left(\alpha_{i j}-\alpha_{j i}\right)\right) .
\end{aligned}
$$


where $\left|\mathcal{N}_{i}\right|$ is the cardinality of $\mathcal{N}_{i}$ (i.e. the number of neighbors of robot $\mathbf{R}_{i}$ ). It is clear that the stochastic operator, as defined in [9]:

$$
\lim _{d t \rightarrow 0} \frac{\mathbb{E}_{\epsilon_{z}}[\Delta V(t)]}{d t}=-4 \cdot \sum_{i=1}^{N} \mathbb{E}_{\epsilon_{z}}\left[\tilde{u}_{i}\right] \sum_{j=1}^{N} a_{i j} e_{i j} \cos \alpha_{i j}
$$

is non-positive. Indeed if the expected value $\mathbb{E}_{\epsilon_{z}}\left[\tilde{u}_{i}\right]$ has the same sign as $u_{i}$ (as stated in condition (ii) of the theorem) we have

$$
\begin{aligned}
\lim _{d t \rightarrow 0} \frac{\mathbb{E}_{\epsilon_{z}}[\Delta V(t)]}{d t} & =-4 \cdot \sum_{i=1}^{N} \mathbb{E}_{\epsilon_{z}}\left[\tilde{u}_{i}\right] \sum_{j=1}^{N} a_{i j} e_{i j} \cos \alpha_{i j} \\
& =-4 \cdot \sum_{i=1}^{N} \frac{\mathbb{E}_{\epsilon_{z}}\left[\tilde{u}_{i}\right] \cdot u_{i}}{k_{u}\left(t, \mathbf{e}_{i}, \boldsymbol{\alpha}_{i}\right)} \\
& \leq 0 .
\end{aligned}
$$

In other words, the sequence of Lyapunov values $\{V(0), V(d t), \ldots, V(n \cdot d t)\}$ is a supermartingale as it satisfies $\mathbb{E}_{\epsilon_{z}}[V((n+1) d t) \mid V(0), \ldots, V(n \cdot d t)] \leq V(n \cdot d t)$ when $d t \rightarrow 0$. Again combining with the Krasovskii-LaSalle principle of the first proof, we conclude that the dynamical system converges to a common point in space.

Figure 3(b) shows a simulation run where four robots converge to a single rendezvous location using the control law defined by Equation 6 on noisy observations:

$$
\left\{\begin{array}{l}
\tilde{u}_{i}=K_{u} \sum_{\mathbf{R}_{j} \in \mathcal{N}_{i}} \tilde{e}_{i j} \cos \tilde{\alpha}_{i j} \\
\tilde{\omega}_{i}=K_{\omega} \sum_{\mathbf{R}_{j} \in \mathcal{N}_{i}} \sin \tilde{\alpha}_{i j}
\end{array} .\right.
$$

In Figure 3(b), the noise component is sampled from a two dimensional Gaussian distribution with a mean at zero and a diagonal covariance matrix (i.e. $\tilde{e}_{i j}$ and $\tilde{\alpha}_{i j}$ are independent), thus it is easy to verify that

$$
\begin{gathered}
\mathbb{E}_{\epsilon_{z}}\left[\tilde{u}_{i}\right]=K_{u} \sum_{\mathbf{R}_{j} \in \mathcal{N}_{i}} \mathbb{E}_{\epsilon_{z}}\left[\tilde{e}_{i j} \cos \tilde{\alpha}_{i j}\right] \\
\stackrel{\text { independence }}{=} K_{u} \sum_{\mathbf{R}_{j} \in \mathcal{N}_{i}} \mathbb{E}_{\epsilon_{z}}\left[\tilde{e}_{i j}\right] \mathbb{E}_{\epsilon_{z}}\left[\cos \tilde{\alpha}_{i j}\right] \\
=K_{u} \sum_{\mathbf{R}_{j} \in \mathcal{N}_{i}} e_{i j} \cos \alpha_{i j}=u_{i}
\end{gathered}
$$

and that $\mathbb{E}_{\epsilon_{z}}\left[\tilde{u}_{i}\right] \cdot u_{i}=u_{i}^{2} \geq 0$. The other conditions were already verified for the deterministic control law in the previous section.

Remark 2: The above theorem is valid as long as the duration of a time step is fairly small with respect to the forward and rotational motion of each robot. In practice, however, observation cycles may be asynchronous and delays may extend the duration of time steps. Fortunately, it is possible to artificially limit the time step duration by enforcing a given control law to ignore old observations and simply stop the robot's motion if no new observation has been made. In Figure 4, we made two simulation runs with identical parameters, except that in Figure 4(b) robots would stop when nothing is observed. In both runs, observations of

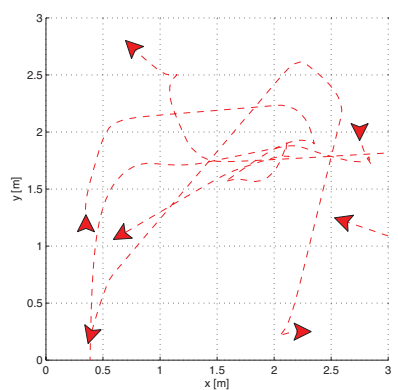

(a)

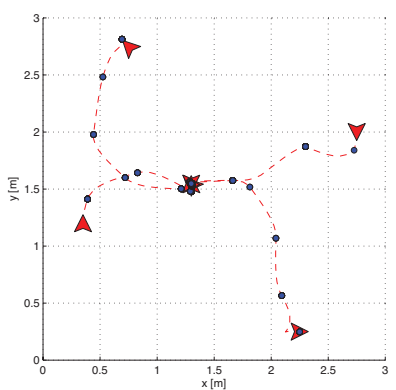

(b)
Fig. 4. Four robots apply the control law of Equation 10 with a large average time step duration, $d t=10[\mathrm{~s}]$. (a) The robots do not take into account the fact that $d t$ is large with respect to control outputs, hence the robots travel using very old observations and are not able to converge. (b) The robots discard observations that are older than 2[s], hence the robots travel shorter distances in-between observations and artificially lower the effective duration of time steps and converge. The blue dots indicate places where the robots stopped because no new observation was received. Here we have used $K_{u}=1 / 2$ and $K_{\omega}=2$. Additionally we have assumed an independent noise $\epsilon_{z}=\left[\epsilon_{e}, \epsilon_{\alpha}\right]^{\top}$ with $\epsilon_{e} \sim \mathcal{N}\left(0,0.05^{2}\left[\mathrm{~m}^{2}\right]\right)$ and $\epsilon_{\alpha} \sim \mathcal{N}\left(0,0.15^{2}\left[\mathrm{rad}^{2}\right]\right)$.

other robots may come asynchronously and may be measured at completely different times. We notice that the stop-andgo behavior implemented in the second figure effectively reduces the travelled distance in-between time steps, thus artificially lowering the time step duration with respect to the control law applied. It shows a successful convergence whereas Figure 4(a) shows robots unable to reach a common meeting point.

\section{BAYESIAN CONTROL}

In practice, it is often beneficial to incorporate the uncertainties of the system. Especially when sensors are noisy and update rates are low, adding a probabilistic dimension to the position of nearby robots may yield to better estimates of their actual position. In other words, although each robot may have a different belief about the position of its neighbors, the group should still be able to rendezvous. In this section, we will explain how a Bayesian approach may be combined with a control law satisfying Theorem 2 to exhibit successful convergence.

\section{A. Bayes Filter}

A given robot $\mathbf{R}_{i}$ needs to track the position of the other agents in the network otherwise known as its neighbors. Hence, for each neighboring robot $\mathbf{R}_{j}$, the robot $\mathbf{R}_{i}$ maintains a belief distribution $\operatorname{bel}\left(\mathbf{x}_{i j}^{(k)}\right)$ of the state $\mathbf{x}_{i j}^{(k)}$ of $\mathbf{R}_{j}$ at each time step $k$ (i.e. $t=k \cdot d t$ ). We can group all state estimates at time step $k$ of neighboring robots in a set $\mathbf{X}_{i}^{(k)}=\left\{\mathbf{x}_{i j}^{(k)} \mid \mathbf{R}_{j} \in \mathcal{N}_{i}\right\}$. The state of a robot defines quantities of interest, in our case they could simply be the relative range and bearing, that is $\mathbf{x}_{i j}^{(k)}=\left[e_{i j}^{(k)}, \alpha_{i j}^{(k)}\right]^{\top}$ where $e_{i j}(k d t) \equiv e_{i j}^{(k)}$ and $\alpha_{i j}(k d t) \equiv \alpha_{i j}^{(k)}$. As explained in [25], the most general algorithm to compute beliefs is the Bayes filter algorithm, which calculates the belief distribution bel with respect the observations and control actions performed assuming that past and future data are independent if one knows the current state $\mathbf{x}_{i j}^{(k)}$. Algorithm 1 
shows the Bayes filter adapted to our problem statement with $\mathbf{u}_{i}^{(k)}$ being a control action, $\mathbf{Z}_{i}^{(k)} \equiv Z_{i}(k d t), \mathbf{z}_{i j}^{(k)} \equiv z_{i j}(k d t)$ and $\eta$ a normalizing constant such that the integral over the belief sums to one. The algorithm as shown here assumes independence of the different measurements $\mathbf{z}_{i j}^{(k)}$, which is reasonable, and uses two probability density functions: $\mathbf{p}\left(\mathbf{x}_{i j}^{(k)} \mid \mathbf{u}_{i}^{(k)}, \mathbf{x}_{i j}^{(k-1)}\right)$, the state transition probability, and $\mathbf{p}\left(\mathbf{z}_{i j}^{(k)} \mid \mathbf{x}_{i j}^{(k)}\right)$, the measurement probability.

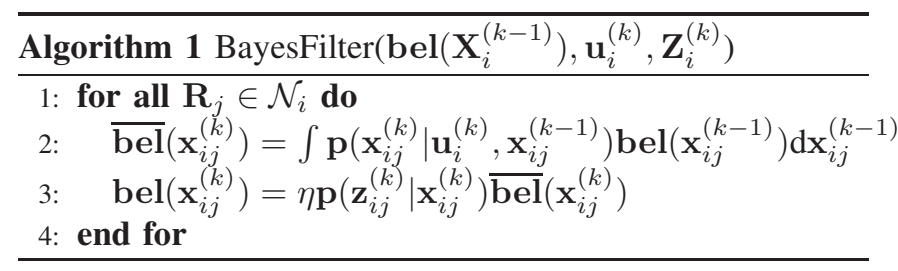

The state transition probability captures how the range and bearing of neighboring robots $\mathbf{R}_{j}$ of $\mathbf{R}_{i}$ are affected by the motion of robot $\mathbf{R}_{i}$ throughout a time step. This probability can integrate potential models about the motion of neighboring robots as well as the coordinate transformation induced by a motion of the robot $\mathbf{R}_{i}$ itself. As an example, let us consider a very basic motion model where we assume to have no knowledge about the motion of nearby robots (i.e. random walk): we can assume that the uncertainty about their relative position propagates according to a zeromean Gaussian distribution with covariance matrix $\Sigma_{m}$. The motion of $\mathbf{R}_{i}$ on the other hand is given by its control law in the form of a forward motion $\hat{u}_{i}$ and rotational motion $\hat{\omega}_{i}$ and we assume no uncertainties there. Using the update Equation 8, we can obtain:

$$
\mathbf{p}\left(\mathbf{x}_{i j}^{(k)} \mid \mathbf{u}_{i}^{(k)}, \mathbf{x}_{i j}^{(k-1)}\right)=\Phi\left(e_{i j}^{(k)}\left[\begin{array}{c}
\cos \left(\alpha_{i j}^{(k)}\right) \\
\sin \left(\alpha_{i j}^{(k)}\right)
\end{array}\right] \mid \mu_{m}, \Sigma_{m}\right)
$$

where $\Phi(x \mid \mu, \Sigma)$ is the multivariate Gaussian probability density function with a mean $\mu$ and covariance matrix $\Sigma$ and

$$
\mu_{m}=\left[\begin{array}{cc}
\cos \left(\hat{\omega}_{i} d t\right) & \sin \left(\hat{\omega}_{i} d t\right) \\
-\sin \left(\hat{\omega}_{i} d t\right) & \cos \left(\hat{\omega}_{i} d t\right)
\end{array}\right]\left[\begin{array}{c}
e_{i j}^{(k)} \cos \left(\alpha_{i j}^{(k)}\right)-\hat{u}_{i} d t \\
e_{i j}^{(k)} \sin \left(\alpha_{i j}^{(k)}\right)
\end{array}\right] .
$$

The measurement probability describes the likelihood of making an observation assuming that the true state of a neighbor is known. As an example, we can simply use the Equation 2 in the form

$$
\mathbf{p}\left(\mathbf{z}_{i j}^{(k)} \mid \mathbf{x}_{i j}^{(k)}\right)=\operatorname{pdf}_{z}\left(\mathbf{z}_{i j}^{(k)}-\mathbf{x}_{i j}^{(k)}\right) .
$$

\section{B. Control Law}

Theorem 3: Given a symmetric and weakly connected group of $N$ differential-wheeled robots $\mathbf{R}_{1}, \ldots, \mathbf{R}_{N}$ driven by the kinematic Equation 1, the probabilistic control law:

$$
\left\{\begin{array}{l}
\hat{u}_{i}=\mathbb{E}_{\mathbf{b e l}}\left[k_{u}\left(t, \mathbf{e}_{i}, \boldsymbol{\alpha}_{i}\right)\right] \sum_{\mathbf{R}_{j} \in \mathcal{N}_{i}} \mathbb{E}_{\mathbf{b e l}}\left[e_{i j} \cos \alpha_{i j}\right] \\
\hat{\omega}_{i}=\mathbb{E}_{\mathbf{b e l}}\left[k_{\omega}\left(t, \mathbf{e}_{i}, \boldsymbol{\alpha}_{i}\right)\right]
\end{array}\right.
$$

where $k_{u}(\cdot)>0$ for all $t \geq 0$, drives the group almost surely to a common rendezvous point if and only if $(i) \hat{u}_{i}=0$ and the expected value $\mathbb{E}_{\text {bel }}\left[e_{i j}\right]$ over the belief is not zero $\left(\mathbb{E}_{\text {bel }}\left[e_{i j}\right] \neq 0\right.$ ) imply that $\mathbb{E}_{\text {bel }}\left[k_{\omega}(\cdot)\right] \neq 0$ for at least one pair of robots $\mathbf{R}_{i}$ and $\mathbf{R}_{j}$ and (ii) the filter used to compute bel is optimal in the sense that the estimation $\mathbb{E}_{\text {bel }}\left[e_{i j} \cos \alpha_{i j}\right]$ is unbiased with respect the observation noise $\left(\mathbb{E}_{\epsilon_{z}}\left[\mathbb{E}_{\mathbf{b e l}}\left[e_{i j} \cos \alpha_{i j}\right]\right]=e_{i j} \cos \alpha_{i j}\right)$ for all robots $\mathbf{R}_{i}$ and $\mathbf{R}_{j} \in \mathcal{N}_{i}$.

Remark 3: The first condition $(i)$ on $k_{\omega}(\cdot)$ simply means that if a robot does not believe that a rendezvous is reached and its forward speed is null, it should have a non-zero rotational speed.

Remark 4: The second condition (ii) is more restrictive than its counterpart in Theorem 2 as it is more convenient to express it in terms of optimality and unbiasness of the filter. Indeed, a more general condition would state that the expected value of $\mathbb{E}_{\epsilon_{z}}\left[\hat{u}_{i}\right]$ over the observation noise should have the same sign than its equivalent deterministic control $u_{i}$ as given by Equation 3 (i.e. $\mathbb{E}_{\epsilon_{z}}\left[\hat{u}_{i}\right] \cdot u_{i} \geq 0$ ), but it is less practical to use.

Proof: The proof follows directly from the proof of Theorem 2. As in Equation 9, we get

$$
\lim _{d t \rightarrow 0} \frac{\mathbb{E}_{\epsilon_{z}}[\Delta V(t)]}{d t} \leq 0
$$

meaning that the sequence of Lyapunov values is a supermartingale. Using the Krasovskii-LaSalle principle, we can conclude that the dynamical system converges to a common meeting point.

Finally, we can transform Equation 6 into a probabilistic control law:

$$
\left\{\begin{array}{l}
\hat{u}_{i}=K_{u} \sum_{\mathbf{R}_{j} \in \mathcal{N}_{i}} \mathbb{E}_{\mathbf{b e l}}\left[e_{i j} \cos \alpha_{i j}\right] \\
\hat{\omega}_{i}=K_{\omega} \sum_{\mathbf{R}_{j} \in \mathcal{N}_{i}} \mathbb{E}_{\mathbf{b e l}}\left[\sin \alpha_{i j}\right]
\end{array} .\right.
$$

Unfortunately, except for simple state transition probabilities, measurement probabilities, and actual observation noise distributions, the convergence properties are often impossible to prove analytically as the equations become intractable. However, it is possible to use numerical approximation and verify that the expected value of the control law satisfies the second condition (ii) of the theorem.

\section{EXPERIMENTS}

\section{A. Real Robots}

Experiments were conducted with four Khepera III robots equipped with a range and bearing module. The noise characteristics of this module are shown in Figure 5 where we can observe that the range and bearing noise behave like independent Gaussian variables with standard deviations of $0.15[\mathrm{~m}]$ and $0.14[\mathrm{rad}]$ respectively. The update frequency of the positioning board is lowered to $0.5 \mathrm{~Hz}$ meaning that a robot $\mathbf{R}_{i}$ makes at most one observation every two seconds for each robot nearby. Three sets of experiments were performed in a $3 \times 3\left[\mathrm{~m}^{2}\right]$ arena:

1) Basic: The robots are controlled by Equation 10 and the stop-and-go behavior explained in Remark 2. As we have 


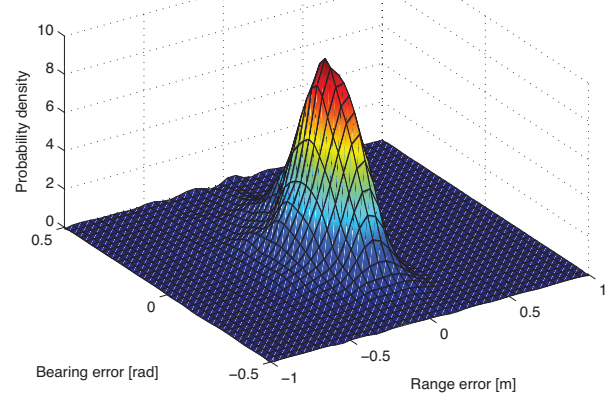

Fig. 5. Empirical probability density function of the noise $\epsilon_{z}$ of the range and bearing module. The covariance matrix of the range and bearing noises is $\Sigma=[0.0221-0.0011 ;-0.00110 .0196]$ which shows little dependency of the random variables.

seen, if the noises affecting the range and the bearing are independent, this control law converges.

2) Motion: The robots are controlled by Equation 10 but use a motion model based on their wheel encoders (odometry) to update the position of the latest observations with respect to their local coordinate frame. In other words, they assume that the position of their neighbors is given by the latest measurement that they continuously update to account for self-locomotion.

3) Bayesian: The robots use Equation 14. A particle filter implements Algorithm 1 with 512 particles per neighbors using Equations 11 and 12. Figure 7(a) shows a run performed by the real robots using this Bayesian control law.

For all sets, $K_{u}=1 / 2$ and $K_{\omega}=2$ and the underlying graph is assumed to be fully connected. The position of each robot is monitored with SwisTrack [15] during 60 seconds. After 10 runs, the root mean square error (RMSE) defined by the Euclidean distance between each pair of robot is computed and displayed in Figure 6(a). Note that the real robots cannot meet at a single point due their size: the minimal distance between two robots is at least equal to the diameter of a robot which is approximately $12[\mathrm{~cm}]$. Although not conclusive, these results show in average a quicker convergence for both approaches taking self-locomotion into account. All approaches show successful convergence and confirm the mathematical properties developed in previous sections.

\section{B. Simulation}

To obtain additional quantitative results we resort to use a kinematic point simulator where the motion of the robots is given by Equation 8 with an update step lasting $d t=64[\mathrm{~ms}]$. The robots have an infinitesimal size, allowing them to meet at the same physical location. Additionally, the noise of the range and bearing observations is sampled from a multivariate Gaussian distribution with a diagonal covariance matrix $\Sigma=\operatorname{diag}\left(\left[\sigma_{e}^{2}, \sigma_{\alpha}^{2}\right]\right)$. All other parameters are set to reflect as closely as possible reality. Although not shown here, setting the real noise ratios into the simulator exhibits near-to identical convergence properties than Figure 6(a). We perform the same three sets of experiments with two observation noise profiles.

First we use a range noise $\sigma_{e}=0.5[\mathrm{~m}]$ and a bearing noise $\sigma_{\alpha}=0.14[\mathrm{rad}]$. After 1000 runs, the RMSE is computed and displayed in Figure 6(b). We can clearly observe the benefits of the Bayesian control law which due to its better estimate of the other robots' position is able to converge faster. As expected, the basic control performs worst.

Second we use a range noise $\sigma_{e}=0.15[\mathrm{~m}]$ and a bearing noise $\sigma_{\alpha}=1[\mathrm{rad}]$. After 1000 runs, the RMSE is computed and displayed in Figure 6(c). Again, the Bayesian control performs best with a faster a more predictable convergence.

\section{Discussion}

The advantages of using a Bayesian approach to solve the rendezvous serves is two-fold. On one hand, it allows us to exploit the knowledge of the sensory noise characteristics and incorporates it nicely in a single unified framework. On the other hand, the motion model (if known) can result in faster convergence times or create interesting behaviors that are hardly possible using the standard reactive rendezvous equation (Equation 7). In the simplistic model explained in Equation 11, we have assumed no information about the motion of neighboring robots. In a real application, this assumption might be too pessimistic since the control laws implemented on all the robots belonging to the team might be engineered and therefore exploitable for further optimization. Therefore, although we have used this simple equation as our state transition probability to allow a fair evaluation of the convergence performances, exploiting this knowledge could yield far better results if carefully chosen. To finish on an upbeat note, Figures 7(b) and 7(c) show that with minimal changes to the control law or the connection graph it is possible to achieve, on real robots with noisy sensors, complex behaviors such as a reconfiguration into a predefined shape or a cyclic pursuit.

\section{CONCLUSION}

In this paper we have proved, via thorough mathematical analysis, sufficient conditions to perform a rendezvous maneuver on a group of differential-wheeled robots endowed with an on-board, noisy, local positioning system. In particular, we extended the simple framework of reactive control with a layer of Bayesian reasoning allowing to solve the rendezvous problem more efficiently and in a probabilistically sound way. Finally, we have performed systematic experiments, both on real robots and simulation, to show the benefits of our Bayesian approach running simplistic update rules. Future work includes the definition of more efficient motion and observation models to merge more complex behaviors.

\section{REFERENCES}

[1] H. Ando, Y. Oasa, I. Suzuki, and M. Yamashita. Distributed memoryless point convergence algorithm for mobile robots with limited visibility. IEEE Transactions on Robotics and Automation, 15(5):818828, 1999.

[2] C. Caicedo-Nunez and M. Zefran. Probabilistic guarantees for rendezvous under noisy measurements. In American Control Conference, 2009, pages 5180-5185, 2009.

[3] M. Cao, A. Morse, and B. Anderson. Reaching a consensus in a dynamically changing environment: A graphical approach. SIAM Journal on Control and Optimization, 47(2):575-601, 2008.

[4] D. Dimarogonas and K. Johansson. Further results on the stability of distance-based multi-robot formations. In American Control Conference, pages 2972-2977, 2009. 


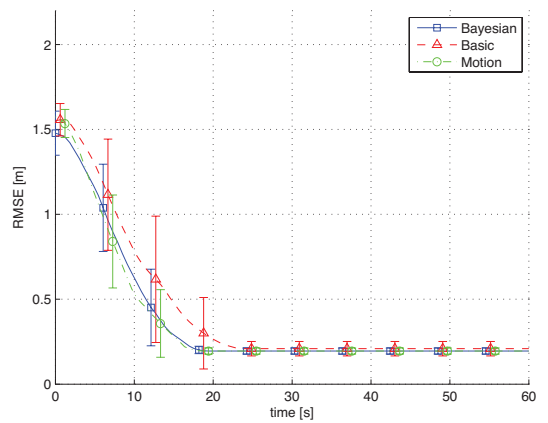

(a)

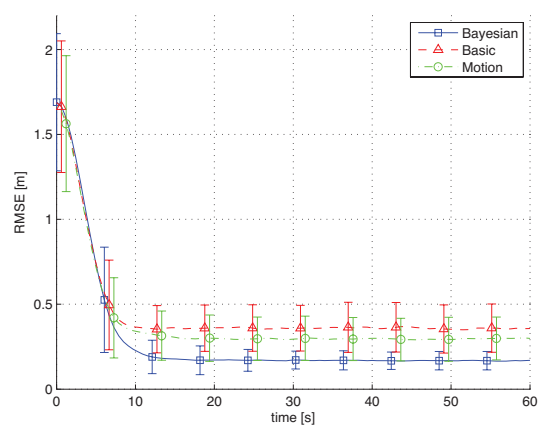

(b)

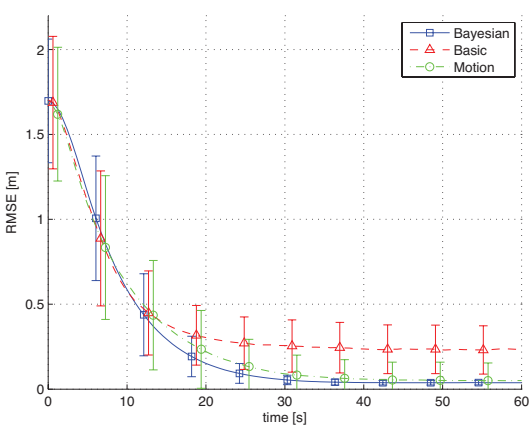

(c)

Fig. 6. Average root mean square distance between each pair of robots over time for (a) the real robots, (b) the simulated robots with a noise $\epsilon_{z}=\left[\epsilon_{e}, \epsilon_{\alpha}\right]^{\top}$ with $\epsilon_{e} \sim \mathcal{N}\left(0,0.5^{2}\left[\mathrm{~m}^{2}\right]\right)$ and $\epsilon_{\alpha} \sim \mathcal{N}\left(0,0.14^{2}\left[\operatorname{rad}^{2}\right]\right)$ and (c) the simulated robots with $\epsilon_{e} \sim \mathcal{N}\left(0,0.15^{2}\left[\mathrm{~m}^{2}\right]\right)$ and $\epsilon_{\alpha} \sim \mathcal{N}\left(0,1^{2}\left[\operatorname{rad}^{2}\right]\right)$. The errorbars show the standard deviation.

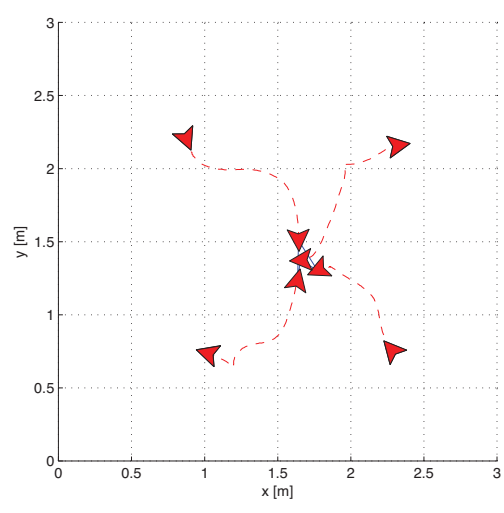

(a)

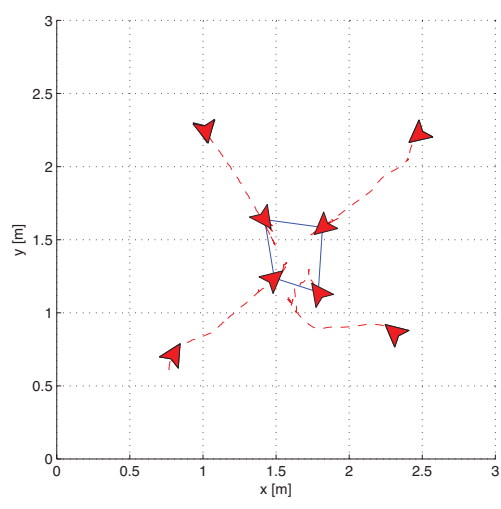

(b)

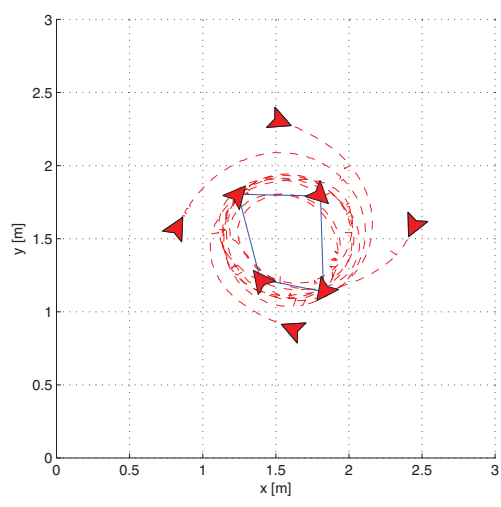

(c)

Fig. 7. Three runs performed on real robots using the Bayesian control law and tracked using SwisTrack. (a) The robots achieve rendez-vous, (b) they create a configuration approaching a square with a diagonal of $0.6[\mathrm{~m}]$ and (c) they perform cyclic pursuit.

[5] D. Dimarogonas and K. Kyriakopoulos. On the rendezvous problem for multiple nonholonomic agents. IEEE Transactions on Automatic Control, 52(5):916-922, 2007.

[6] E. Eisenberg and D. Gale. Consensus of subjective probabilities: The pari-mutuel method. The Annals of Mathematical Statistics, 30(1): 165-168, 1959.

[7] R. Falconi, S. Gowal, J. Pugh, and A. Martinoli. Graph-based distributed control for non-holonomic vehicles engaged in a reconfiguration task using local positioning information. In Conference on Robot Communication and Coordination, 2009. doi: 10.4108/ICST. ROBOCOMM2009.5865.

[8] M. Ji and M. Egerstedt. Distributed coordination control of multiagent systems while preserving connectedness. IEEE Transactions on Robotics, 23(4):693-703, 2007.

[9] H. J. Kushner. Stochastic stability and control. Academic Press New York, 1967. ISBN 0124301509.

[10] G. Lafferriere, J. Caughman, and A. Williams. Graph theoretic methods in the stability of vehicle formations. In Proceedings of the American Control Conference, volume 4, pages 3729-3734, 2004.

[11] J. Lawton, R. Beard, and B. Young. A decentralized approach to formation maneuvers. IEEE Transactions on Robotics and Automation, 19(6):933-941, 2003.

[12] N. Leonard and E. Fiorelli. Virtual leaders, artificial potentials and coordinated control of groups. In IEEE Conference on Decision and Control, volume 3, pages 2968-2973, 2001.

[13] J. Lin, A. Morse, and B. Anderson. The multi-agent rendezvous problem. In Proceedings of the IEEE Conference on Decision and Control, volume 2, pages 1508-1513, 2003.

[14] J. Lin, A. Morse, and B. Anderson. The multi-agent rendezvous problem - the asynchronous case. In Proceedings of the IEEE Conference on Decision and Control, volume 2, pages 1926-1931, 2004.

[15] T. Lochmatter, P. Roduit, C. Cianci, N. Correll, J. Jacot, and A. Martinoli. Swistrack - a flexible open source tracking software for multi- agent systems. In IEEE/RSJ International Conference on Intelligent Robots and Systems, pages 4004-4010, 22-26 2008.

[16] R. Olfati-Saber, J. Fax, and R. Murray. Consensus and cooperation in networked multi-agent systems. Proceedings of the IEEE, 95(1): 215-233, 2007.

[17] A. Prorok, A. Arfire, A. Bahr, J. Farserotu, and A. Martinoli. Indoor navigation research with the khepera iii mobile robot: An experimental baseline with a case-study on ultra-wideband positioning. In $2010 \mathrm{In}$ ternational Conference on Indoor Positioning and Indoor Navigation, 2010. doi: 10.1109/IPIN.2010.5647880.

[18] J. Pugh, X. Raemy, C. Favre, R. Falconi, and A. Martinoli. A fast onboard relative positioning module for multirobot systems. IEEE/ASME Transactions on Mechatronics, 14(2):152-162, 2009.

[19] W. Ren and R. Beard. Distributed Consensus in Multi-vehicle Cooperative Control: Theory and Applications. Springer Publishing Company, Incorporated, 2007. ISBN 1848000146, 9781848000148.

[20] C. Reynolds. Flocks, herds and schools: A distributed behavioral model. SIGGRAPH Computer Graphics, 21(4):25-34, 1987.

[21] A. Sinha and D. Ghose. Generalization of linear cyclic pursuit with application to rendezvous of multiple autonomous agents. IEEE Transactions on Automatic Control, 51(11):1819-1824, 2006.

[22] B. Smith, J. Wang, and M. Egerstedt. Persistent formation control of multi-robot networks. In IEEE Conference on Decision and Control, pages 471-476, 2008.

[23] B. Smith, J. Wang, M. Egerstedt, and A. Howard. Automatic formation deployment of decentralized heterogeneous multi-robot networks with limited sensing capabilities. In IEEE International Conference on Robotics and Automation, pages 730-735, 2009.

[24] S. Smith, M. Broucke, and B. Francis. Curve shortening and the rendezvous problem for mobile autonomous robots. IEEE Transactions on Automatic Control, 52(6):1154-1159, 2007.

[25] S. Thrun, W. Burgard, and D. Fox. Probabilistic Robotics (Intelligent Robotics and Autonomous Agents). The MIT Press, 2005. ISBN 0262201623 . 